\title{
A POWER MEAN INEQUALITY FOR THE GRÖTZSCH RING FUNCTION
}

\author{
Gendi Wang, XiaOhui Zhang And Yuming Chu
}

\begin{abstract}
The Grötzsch ring function has numerous applications in geometric function theory and its properties have been investigated by many authors. Here we extend an earlier functional inequality involving the Grötzsch ring function and the geometric mean, due to Anderson, Vamanamurthy and Vuorinen, to the case of power mean.
\end{abstract}

Mathematics subject classification (2010): 30C62, 33E05.

Keywords and phrases: Grötzsch ring function, power mean, inequality.

\section{REFERENCES}

[1] G. D. Anderson, S.-L. QiU, M. K. Vamanamurthy, Grötzsch ring and quasiconformal distortion functions, Hokkaido J. Math., 24 (1995), 551-566.

[2] G. D. Anderson, S.-L. Qiu, M. K. Vamanamurthy, M. Vuorinen, Generalized elliptic integrals and modular equations, Pacific J. Math., 192 (2000), 1-37.

[3] G. D. Anderson, M. K. Vamanamurthy, M. Vuorinen, Special functions of quasiconformal theory, Exposition Math., 7 (1989), 97-138.

[4] G. D. Anderson, M. K. Vamanamurthy, M. Vuorinen, Functional inequalities for complete elliptic integrals and their ratios, SIAM J. Math. Anal., 21 (1990), 536-549.

[5] G. D. Anderson, M. K. Vamanamurthy, M. Vuorinen, Inequalities for plane quasiconformal mappings, in: Contemp. Math., vol. 169, Amer. Math. Soc., 1994, pp. 1-27.

[6] G. D. Anderson, M. K. Vamanamurthy, M. Vuorinen, Conformal Invariants, Inequalities, and Quasiconformal Maps, John Wiley \& Sons, New York, 1997.

[7] R. Balasubramanian, S. Ponnusamy, M. Vuorinen, Functional inequalities for the quotients of hypergeometric functions, J. Math. Anal. Appl., 218 (1998), 256-268.

[8] F. Bowman, Introduction to Elliptic Functions with Applications, Dover, New York, 1961.

[9] P. S. Bullen, Handbook of Means and Their Inequalities, Mathematics and Its Applications, vol. 560, Kluwer Academic Publishers, Dordrecht, 2003.

[10] P. F. B YRD, M. D. FrIEDMAN, Handbook of Elliptic integrals for Engineers and Physicists, 2nd ed., Die Grundlehren Math. Wiss., 67, Springer-Verlag, Berlin, 1971.

[11] G. Hardy, J. E. Littlewood, G. Pólya, Inequalities, Cambridge Univ. Press, 1952.

[12] R. KÜHNAU, The conformal module of quadrilaterals and of rings, in: R. Kühnau(Ed.), Handbook of Complex Analysis: Geometric Function Theory, vol. 2, Elsevier, 2005, pp. 99-129.

[13] O. Lehto, K. I. VirTAnen, Quasiconformal mappings in the plane, Springer-Verlag, New York, 1973.

[14] S. L. QIU, Grötzsch ring and Ramanujan's modular equations, Acta Math. Sinica, 43 (2000), 283290.

[15] S.-L. QIU, M. VUORINEN, Infinite products and normalized quotients of hypergeometric functions, SIAM J. Math. Anal., 30 (1999), 1057-1075.

[16] A. VASILEV, Moduli of Families of Curves for Conformal and Quasiconformal Mappings, Lecture Notes in Math., vol.1788, Springer-Verlag, Berlin, 2002.

[17] M. K. Vamanamurthy, M. Vuorinen, Functional inequalities, Jacobi products and quasiconformal maps, Illinois J. Math., 38 (1994), 394-419.

[18] M. VuORINEN, Conformal geometry and quasiregular mappings, Lecture notes in Mathematics 1319, Springer-Verlag, Berlin, 1988. 
[19] M. Vuorinen, Singular values, Ramanujan's modular equations, and Landen transformations, Studia Math., 121 (1996), 221-230. 